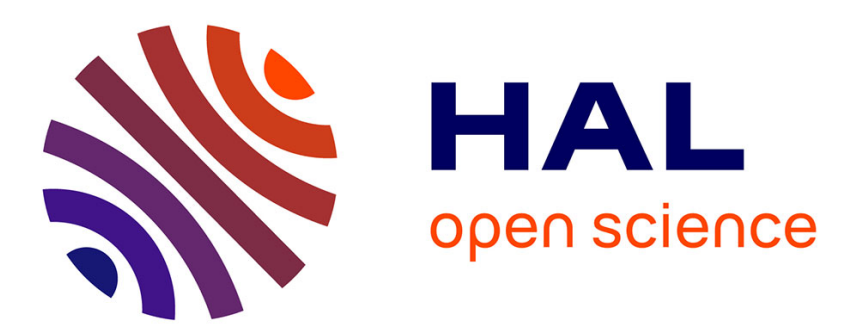

\title{
A physical model of the photo- and radiation-induced degradation of ytterbium-doped silica optical fibres
}

\author{
Franck Mady, Jean-Bernard Duchez, Yasmine Mebrouk, Mourad
}

Benabdesselam

\section{- To cite this version:}

Franck Mady, Jean-Bernard Duchez, Yasmine Mebrouk, Mourad Benabdesselam. A physical model of the photo- and radiation-induced degradation of ytterbium-doped silica optical fibres. AIP Conference Proceedings, 2014, 10.1063/1.4900462 . hal-01223816

\section{HAL Id: hal-01223816 \\ https://hal.science/hal-01223816}

Submitted on 3 Nov 2015

HAL is a multi-disciplinary open access archive for the deposit and dissemination of scientific research documents, whether they are published or not. The documents may come from teaching and research institutions in France or abroad, or from public or private research centers.
L'archive ouverte pluridisciplinaire HAL, est destinée au dépôt et à la diffusion de documents scientifiques de niveau recherche, publiés ou non, émanant des établissements d'enseignement et de recherche français ou étrangers, des laboratoires publics ou privés. 


\title{
A Physical Model of the Photo- and Radiation-induced Degradation of Ytterbium-doped Silica Optical Fibres
}

\author{
Franck Mady ${ }^{\text {a)}}$, Jean-Bernard Duchez, Yasmine Mebrouk, \\ and Mourad Benabdesselam
}

University of Nice Sophia Antipolis, Laboratoire de Physique de la Matière Condensée (LPMC), CNRS UMR 7336, Parc Valrose, 06108 Nice cedex 2, France

${ }^{a)}$ Corresponding author: franck.mady@unice.fr

\begin{abstract}
We propose a model to describe the photo- or/and the radiation-induced darkening of ytterbium-doped silica optical fibers. This model accounts for the well-established experimental features of photo-darkening. Degradation behaviors predicted for fibers pumped in harsh environments are also fully confirmed by experimental data reported in the work by Duchez et al. (this proceeding), which gives a detailed characterization of the interplay between the effects of the pump and those of a superimposed ionizing irradiation (actual operation conditions in space-based applications for instance). In particular, dependences of the darkening build-up on the pump power, the total ionizing dose and the dose rate are all correctly reproduced. The presented model is a 'sufficient' one, including the minimal physical ingredients required to reproduce experimental features. Refinements could be proposed to improve, e.g., quantitative kinetics.
\end{abstract}

\section{INTRODUCTION}

Ytterbium-doped silica optical fibers (YDF) can be damaged by pump photons (photo-darkening, PD) [1-6] and possibly by external ionizing radiation (radiation-induced darkening, RD) [7-9]. In both cases, darkening results from a large excess optical absorption that develops from ultra-violet up to near infra-red ranges. YDF are nevertheless considered for some critical applications concerned with harsh environments as, e.g., space-based applications, because YDF-based amplifiers allow the design of low size, low weight, high efficiency, high-output power laser sources. In such operation conditions, pump and external radiations will act simultaneously and YDF will be submitted to both darkening types at once. This obviously raises the question of possible superimposition effects: do PD and RD interact? Is the interplay constructive (additive darkening) or destructive (mutual bleaching)?

Although crucial, this superimposition has not been investigated to date. A very original and detailed characterization of the simultaneous photo- and radiation-induced darkening (hereafter abbreviated to PRD) is proposed, in this conference proceeding, by J-B. Duchez et al. The present complementary work presents a model based on the minimal physical mechanisms (free of any semi-empirical approach) that are shown to be sufficient to reproduce all of the experimental features reported in literature for photo-darkening (PD), and in the Duchez's work for radiation-induced darkening (RD) and PRD. Refinements could be proposed to improve quantitative kinetics. The model correctly accounts for the total ionizing dose, dose rate and pump power dependences on the degradation build-up. Such an agreement between a rather wide variety of data and calculated behaviors strongly supports the model and has important consequences in the assessment of the YDF resistance against PD, RD and PRD. Even if PD and RD mitigation routes exist (phosphorus co-doping for PD [10] or cerium co-doping for PD and RD [11,12]), our work sets a novel and proper understanding framework that goes far beyond these empirical recipes. 


\section{MODEL}

The physical processes of PD and RD build-ups are still rather controversial $[13,14]$, but the experimental validation of the present model may help in putting an end to the main controversies. In [7], we concluded that RD of X-ray irradiated YDF originates from the reduction of $\mathrm{Yb}^{3+}$ ions, giving $\mathrm{Yb}^{2+}$ ions and trapped holes. We also highlighted that trapped-hole centers related to PD are identical to those produced by RD. At last, we showed that the thermal bleaching of $\mathrm{PD}$ and $\mathrm{RD}$ is due to hole release and recombination to $\mathrm{Yb}^{2+}$ ions. These findings are consistent with proposals by other teams [2,15-17], notably the Engholm's [15-17] according to which PD is due to an $\mathrm{Yb}^{3+}$ to $\mathrm{Yb}^{2+}$ conversion, again with hole trapping, upon absorption of a UV photon (resulting from the deexcitation of several excited $\mathrm{Yb}^{3+}$ ions) into the charge transfer (CT) bands of $\mathrm{Yb}^{3+}$ ions. Darkening can be bleached by photons at $3.49 \mathrm{eV}(355 \mathrm{~nm})$ [1], $2.28 \mathrm{eV}(543 \mathrm{~nm})$ [2], $1.36 \mathrm{eV}(915 \mathrm{~nm})$ [3], and 1,27 eV (980 nm, Duchez's work). If photo-bleaching (PB) results from the photo-ionization (PI) of trapped holes, this variety of energies can suggest that PI takes place between localized trapped-hole states in the glass band-gap and a continuum, namely the valence band (VB). Then, trapped-hole states responsible for the induced optical loss, i.e. acting as color centers, may spread from at least $\sim 1.27 \mathrm{eV}(980 \mathrm{~nm}$ ) above the VB (consistent with $[18,19])$. Based on these hypotheses, we propose to describe PD, RD and PRD by the model of Fig. 1, which is cast into the form of an energy level scheme.

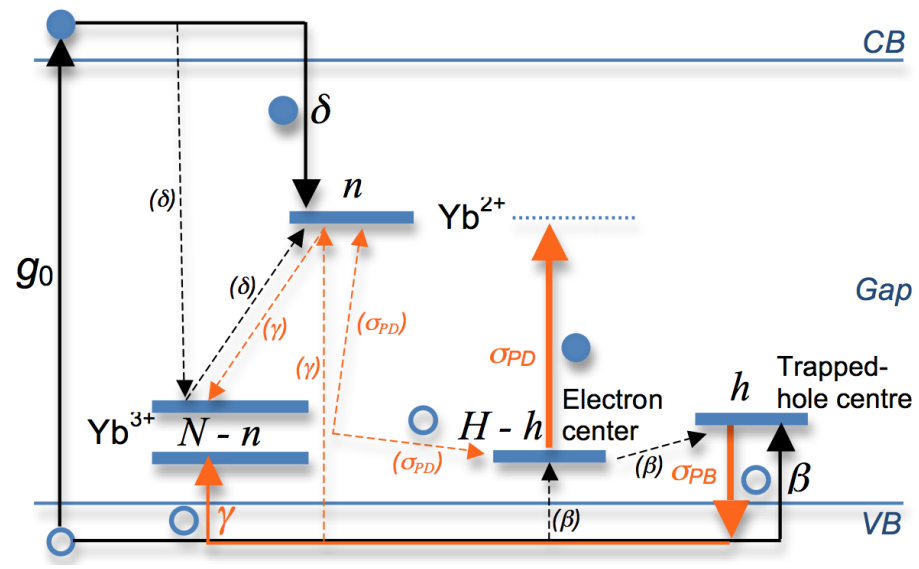

FIGURE 1. Energy level scheme of the proposed model. RD transitions in black, PD and PB ones in orange. Thick arrows $=$ actual energy transitions between initial and final states. Dashed arrows = ionization processes or trapping/recombination steps

The ionizing radiation, responsible for $\mathrm{RD}$, creates electron-hole pairs at a rate $g_{0}$, representative of the dose rate. The total ionizing dose is thus given by $g_{0} \times t_{\text {irr }}$, where $t_{\text {irr }}$ is the irradiation time. It corresponds to the total concentration of electron-hole pairs generated by irradiation. Electrons injected in the conduction band (CB) can be trapped by $\mathrm{Yb}^{3+}$ ions (initial density $N$ ) to form $\mathrm{Yb}^{2+}$ ions in density $n$ ( $\delta$ transitions). Holes in the VB are trapped by gap states to form trapped-hole color centers ( $H$ states occupied by $h$ holes, $\beta$ transitions). This minimal model, which only aims at a qualitative demonstration, considers a single hole trapping level. It also neglects other possible electron traps besides $\mathrm{Yb}^{3+}$ ions as well as the thermal release of carriers. Of course, it would be more realistic to include a distribution of gap levels, especially for hole traps whose depths are a priori dispersed due to the variety of hole centers that have been indentified in silicate or alumino-silicate glasses and to the in-homogeneity of such amorphous materials. These refinements would be required to reproduce actual kinetics accurately, but they would involve further hypotheses as regards, notably, the nature of additional traps and their density of states in the gap. This would introduce a significant complexity that would mud the waters concerning the identification of the crucial mechanisms that are basically sufficient to explain the observed behaviors.

The pump is responsible for the direct $\mathrm{CT}$ of holes from $\mathrm{Yb}^{3+}$ ions to traps, as shown by the $\sigma_{\mathrm{PD}}$ transitions. It thus directly contributes to the increase of the density $h$ of color centers. According to our previous experimental findings [7], we make the basic assumption that hole traps populated by PD are the same as those involved in RD, i.e. filled via the $\beta$ transition. Following Engholm and Norin [16], the higher energy $\mathrm{CT}$ of holes from $\mathrm{Yb}^{3+}$ ions to the VB is neglected. This transition probably exists, but it uses a higher energy and is therefore much less probable. The ionization energy of PD transitions is indeed supplied by the cooperative de-excitation of several excited $\mathrm{Yb}^{3+}$ ions. The rate of this process decreases very rapidly with the number of involved ions, so with the required energy. 
PI of trapped-hole centers corresponds to the $\sigma_{\mathrm{PB}}$ transition. Released holes can recombine to $\mathrm{Yb}^{2+}$ ions $(\gamma$ transitions). In [8], experiments on the thermal bleaching of RD allowed us to establish that the main hole trap involved in $\mathrm{RD}$, and so in $\mathrm{PD}$, was associated with a thermoluminescence glow peak, together with a thermal bleaching peak, in the $400-450{ }^{\circ} \mathrm{C}$ range when heating up at a $1{ }^{\circ} \mathrm{C} \mathrm{s}^{-1}$ constant rate. The kinetic analysis of this glow peak indicated that the thermal activation energy of the related trapped-hole center is about $1.3 \mathrm{eV}$. This estimate is in fair agreement with activation energies obtained by other methods for the thermal bleaching of PD, namely 1.3 $\mathrm{eV}$ [18] and 1.26-1.50 eV [19]. In view of these values, the PI of trapped holes should be most efficiently achieved by photons around $2.5 \mathrm{eV}$ (or more). As a result, it is rather unlikely that PI (and so PB) is directly induced by a single pump photon at $980 \mathrm{~nm}(1.27 \mathrm{eV})$. We therefore propose that the energy required for PI is primarily taken from the de-excitation of $k=2$ or 3 excited $\mathrm{Yb}^{3+}$ ions to reach a significant PI cross-section. This hypothesis is consistent with the recent finding by Jetschke et al. [6] and Li et al. [20] who showed that $\mathrm{Yb}^{3+}$ ions in excited state transfer energy to color centers. As regards $\mathrm{PD}$, we consider that 3 cooperative de-excitations of $\mathrm{Yb}^{3+}$ ions are necessary to reach the first $\mathrm{CT}$ absorption band [16].

Both $\mathrm{Yb}^{2+}$ ions $(n)$ and color centers $(h)$ may contribute to the excess loss in their respective absorption bands. Transient populations in bands are always small so $n$ and $h$ are virtually identical. The excess absorption coefficient $\Delta \alpha$ is as usual proportional to the density of color centers, i.e. to $h \approx n$, which is the quantity to be compared with the measured $\Delta \alpha$. Transitions of Fig. 1 can be rationalized by the set of coupled non-linear differential equations (1)-(4). These equations can then be solved numerically to provide time-resolved PD, RD or PRD.

$$
\begin{gathered}
\frac{d n_{\mathrm{c}}}{d t}=g_{0}-\delta n_{\mathrm{c}}(N-n), \\
\frac{d n}{d t}=\delta n_{\mathrm{c}}(N-n)+\sigma_{\mathrm{PD}} \frac{x_{\text {inv }}^{4}(N-n)^{4}}{\tau^{3}}(H-h)-\gamma h_{\mathrm{v}} n, \\
\frac{d h}{d t}=\beta h_{\mathrm{v}}(H-h)+\sigma_{\mathrm{PD}} \frac{x_{\text {inv }}^{4}(N-n)^{4}}{\tau^{3}}(H-h)-\sigma_{\mathrm{PB}} \frac{x_{\mathrm{inv}}^{k}(N-n)^{k}}{\tau^{k}} N_{\mathrm{v}} h, \\
\frac{d h_{\mathrm{v}}}{d t}=g_{0}-\beta h_{\mathrm{v}}(H-h)+\sigma_{\mathrm{PB}} \frac{x_{\mathrm{inv}}^{k}(N-n)^{k}}{\tau^{k}} N_{\mathrm{v}} h-\gamma h_{\mathrm{v}} n .
\end{gathered}
$$

In (1)-(4), $n_{\mathrm{c}}$ and $h_{\mathrm{v}}$ are the densities of electrons in the CB and of holes in the VB, respectively. $x_{\text {inv }}$ and $\tau$ are the rate of inversion and the non-radiative lifetime of $\mathrm{Yb}^{3+}$ ions. The pump power is varied in the model through $x_{\text {inv }} . N_{\mathrm{V}}$ is the equivalent density of states in the $\mathrm{VB}$. The rate of cooperative de-excitation of the 3 excited $\mathrm{Yb}^{3+}$ ions required for PD is taken proportional to $f_{\mathrm{c}}=\left[x_{\mathrm{inv}}(N-n) / \tau\right]^{3}$. The energy released in this way has to be absorbed by a fourth excited ion to trigger the CT. The probability of this additional step is proportional to the density of available excited ions, i.e. to $x_{\text {inv }}(N-n)$. The latter term is multiplied by $f_{\mathrm{c}}$ to give the overall PD rate corresponding to the fourth power term in equations (2) and (3). The $k$ th power PB term in equations (3) and (4), where $k=2$ or 3, has a similar form as $f_{\mathrm{c}}$. Rigorously, the lifetime of $\mathrm{Yb}^{3+}$ ions is experimentally found to decrease all along the darkening build-up [6,20]. This dependence of $\tau$ on $h$ is not considered here, thus offering a further refinement opportunity. The excitation rate $g_{0}$ is in unit of $\mathrm{cm}^{-3} \mathrm{~s}^{-1}$. The trapping and recombination coefficients $\beta, \delta$ and $\gamma$ are in $\mathrm{cm}^{3} \mathrm{~s}^{-1}$ (product of a cross-section in $\mathrm{cm}^{2}$ by a thermal velocity at the $\mathrm{CB}$ or VB edge in $\mathrm{cm} \mathrm{s}^{-1}$ ). The $\sigma_{\mathrm{PD}}$ and $\sigma_{\mathrm{PB}}$ coefficients serve as proportionality factors for the above detailed probability terms. Although they necessarily include cross-sections (absorption in the CT band or PI cross-section), they do not directly correspond to a cross-section in $\mathrm{cm}^{2} . \sigma_{\mathrm{PD}}$ is actually in $\mathrm{cm}^{12} \mathrm{~s}^{2}$ and $\sigma_{\mathrm{PB}}$ is in $\mathrm{cm}^{3(\mathrm{k}+1)} \mathrm{s}^{\mathrm{k}-1}$. The treatment of equations (1)-(4) was done based on a dimensionless formulation in order to free the discussion from any particular value of the physical parameters and also to highlight a set of a few dimensionless parameter ratios that govern the solutions of the system. Exact stationary solutions have been calculated formally. Time-resolved transient evolutions have been computed by the mean of a numerical resolution of equations (1)-(4) thanks to the Scilab® freeware.

\section{RESULTS}

Because of the presence of a PB mechanism competing with PD and RD, simulated PD and PRD levels always reach a stationary value at long irradiation time, in agreement with data (see [3] for pure PD and the Duchez's article in this proceeding for PRD). A detailed analysis of the equations shows that solutions for pure RD (absence of 
pump: $x_{\mathrm{inv}}=0$ ) are determined by the three following ratios: $r=N / H, \alpha=\beta / \gamma, \theta=\delta / \gamma$, representative of the relative concentration of $\mathrm{Yb}^{3+}$ ions with respect to that of hole traps, of the competition between trapping and recombination of holes and between the formation and recombination of $\mathrm{Yb}^{2+}$ ions due to the ionizing radiation, respectively. The stationary level of pure $\mathrm{PD}\left(g_{0}=0\right)$ is notably controlled by the inversion rate $x_{\mathrm{inv}}$ and the ratio $\sigma_{\mathrm{PD}} / \sigma_{\mathrm{PB}}$.

\section{Model validation for pure PD}

The model was first checked against literature data in case of pure PD, i.e. for $g_{0}=0$. The point was to highlight whether the model is capable of reproducing the well-known experimental features of PD, as reported for instance by Jetschke et al. [3,5]. Two main properties have to be accounted for: $(i)$ the linear dependence of the PD equilibrium level (PDEL) on $x_{\text {inv }}$ [3], and (ii) the power-law dependence on $x_{\text {inv }}$ of the rate constant extracted from the fit of a stretched exponential function to the PD and PB kinetics [3,5]. Both properties have been experimentally established outside of any saturation of the PD level (the PDEL results from equilibrium between PD and PB and not from a saturation effect. It was indeed shown to increase with inversion [3]). We thus deal with situations where traps are not saturated. If there are more $\mathrm{Yb}^{3+}$ ions than hole traps (case $N>H$ or $r>1$ ), the latter will saturate before ytterbium ions (electron traps). At saturation, the hole trap occupancy rate will be $h / H=1$ but, since $h$ and $n$ are virtually equal, the $\mathrm{Yb}^{3+}$ to $\mathrm{Yb}^{2+}$ conversion rate will be $n / N=1 / r<1$. If hole traps are on the contrary more abundant (case $H>N$, i.e. $r<1$ ), saturation will be reached in the conversion of $\mathrm{Yb}^{3+}$ ions first, for $n / N=1$ and so $h / H=\mathrm{r}<1$. The non-saturation of electron and hole traps is thus ensured if $n / N<\min (1,1 / r)$ or, equivalently, if $h / H$ $<<\min (1, r)$. When this condition is fulfilled, several physical cases are found to result in the proportionality between the PDEL and inversion, according to the values of $k, r$ and $\alpha$.

If PI involves 2 excited $\mathrm{Yb}^{3+}$ ions $(k=2)$, the linear relationship between the PDEL and $x_{\text {inv }}$ is given by equation (5) where the PDEL is represented by the steady-state hole trap filling reached under continuous pumping, $(h / H)_{\infty}$, or by the stationary conversion rate of $\mathrm{Yb}^{3+}$ ions, $(n / N)_{\infty}$ :

$$
\left(\frac{h}{H}\right)_{\infty}=r\left(\frac{n}{N}\right)_{\infty}=\left(\frac{\alpha N^{2}}{N_{\mathrm{v}} r^{2} \tau} \frac{\sigma_{\mathrm{PD}}}{\sigma_{\mathrm{PB}}}\right)^{\frac{1}{2}} \times x_{\mathrm{inv}} .
$$

This equation is valid for $(h / H)_{\infty}<<\alpha$, i.e. $(n / N)_{\infty}<<\alpha / r$. Below saturation, this is the case for $\alpha>r$ if $\mathrm{r}<1$ and for $\alpha \geq 1$ if $r \geq 1$. If PI involves 3 excited ions $(k=3)$, the PDEL increases linearly with $x_{\text {inv }}$ if $\alpha<<(h / H)_{\infty}<<$ $\min (1, r)$, i.e. $\alpha / r<<(n / N)_{\infty}<<\min (1,1 / r)$. Then:

$$
\left(\frac{h}{H}\right)_{\infty}=r\left(\frac{n}{N}\right)_{\infty}=\frac{N}{N_{\mathrm{v}} r} \frac{\sigma_{\mathrm{PD}}}{\sigma_{\mathrm{PB}}} \times x_{\mathrm{inv}} .
$$

This situation only exists if $\alpha \leq 1$ (hole trapping cross-section smaller than the hole recombination cross-section). Both equations (5) and (6) imply a linear dependence of the PDEL on the ytterbium concentration $N$. To help in deciding which of these 2 cases most likely corresponds to the actual one, the dose rate dependence of RD in YDF should be experimentally characterized because small $\alpha$ values, which rather correspond to the case of equation (6) for $k=3$, generate much higher dose rate effects than cases with $\alpha \geq 1$ [21] for which the proportionality between the PDEL and $x_{\text {inv }}$ is primarily obtained for $k=2$ according to equation (5).

We also checked that the model predicts a power-law dependence of the fitted rate constant. As done in [3,5] on experimental data, we fitted the transient decays of the calculated absorption coefficient accompanying the PD build-up with a stretched exponential law to extract the rate constant. Numerous tests were done based on different sets of parameters. The empirical stretched exponential law correctly described all of the simulated PD curves. Extracted rate constants were then plotted as a function of the inversion rate $x_{\text {inv }}$; we found a theoretical dependence in $x_{\text {inv }}{ }^{3}$. Such a cubic variation was established for $k=2$ in conditions of equation (5), but also for $k=3$ in conditions of equation (6). This finding is perfectly similar to the experimental relationship established in [3]. It is worth to be noted that the cubic dependence appears as a natural consequence of the model, especially in the case $k=2$ where the basic equations (1)-(4) do not contain any third power term. The power-law dependence of the rate constant and its physical origin will be presented and discussed in detail in a forthcoming publication. 


\section{Model validation for PRD}

Further validation of the model was sought by comparing its predictions, in terms of time-resolved PRD, to the experimental characterizations presented by Duchez et al. (in this proceeding). To allow straightforward comparison, simulations were conducted so as to reproduce experimental protocols. The influence of the total dose, dose rate and pump power, as generated inherently by the model, were thus examined in the same way as in experiments.

The first test consisted in calculating PRD at increasing doses before leaving the system to the sole action of the pump. The results are shown in Fig. 2, which should be compared with its empirical twin (Fig. 2 in the Duchez's article). After the targeted dose was reached, $g_{0}$ was forced to zero to simulate the end of irradiation, but the pump was not turned off (the value of $x_{\text {inv }}$ was maintained throughout the simulation). In this illustrative example, calculations were made for $k=2, \alpha=1$ and $r=1$, in conditions where equation (5) holds, i.e. in the linear regime existing below trap saturation. Other determining dimensionless parameters were: $\theta=1, x_{\text {inv }}=0.2, g_{0} /(\beta N)^{2}=10^{-15}$ (reduced dose rate), $N^{2} \sigma_{\mathrm{PD}} /\left(\tau N_{\mathrm{v}} \sigma_{\mathrm{PB}}\right)=1$ and $N^{3} \sigma_{\mathrm{PD}} /\left(\beta \tau^{3}\right)=2.56 \times 10^{-14}$. Any combination of physical parameters leading to these values of the dimensionless ratios give identical transient and steady-state trap occupancies. We checked that varying these values across decades does not change the properties demonstrated here: degradation kinetics and levels are of course affected, but the general behavior is basically the same. The reduced dose $D$ indicated on each plot is the ratio of the density of electron-hole pairs created by the ionizing dose, $g_{0} \times t_{\text {irr }}$, to the density of $\mathrm{Yb}^{3+}$ ions, $N$. The dimensionless numerical treatment gives the hole trap occupancy $(h / H)$; the latter was converted into the normalized fiber transmission $T$, as reported on the ordinate of Fig. 2, by using $T=\exp \left[-\sigma_{\mathrm{abs}} H L \times(h / H)\right]$, with $\sigma_{\mathrm{abs}} H L=20$. This value was chosen to get a correct transmission scaling with respect to data at $633 \mathrm{~nm}$ (see the Duchez's article), the order of magnitude being quite plausible for a sample length $L=2 \mathrm{~cm}$. Time has been left in dimensionless form on the abscissa. It is measured in units of $(\beta H)^{-1}$, i.e. in units of the minimum trapping lifetime of holes in the VB $\left(\beta H\right.$ in s$^{-1}$ is the maximum trapping rate of holes, obtained when the density of available trapping states is maximal, equal to $H$ ).

Plots of Fig. 2 are very similar to those displayed in its experimental counterpart (Fig. 2 in the Duchez's article). They both highlight the crucial role of the PDEL by showing that the PD or PB action of the pump after PRD depends on the ionizing dose! The pump is responsible for PD as long as the dose has not degraded the system below the PDEL. Otherwise it leads to PB. In all cases, the degradation tends towards the PDEL under the continuous action of the pump after the end of the ionizing irradiation. This effect is obtained because of the crucial assumption made in the model that the same hole traps are shared by PD and RD and, therefore, that the pump is capable of bleaching both PD and RD. This hypothesis is obviously and strongly supported by experimental results.
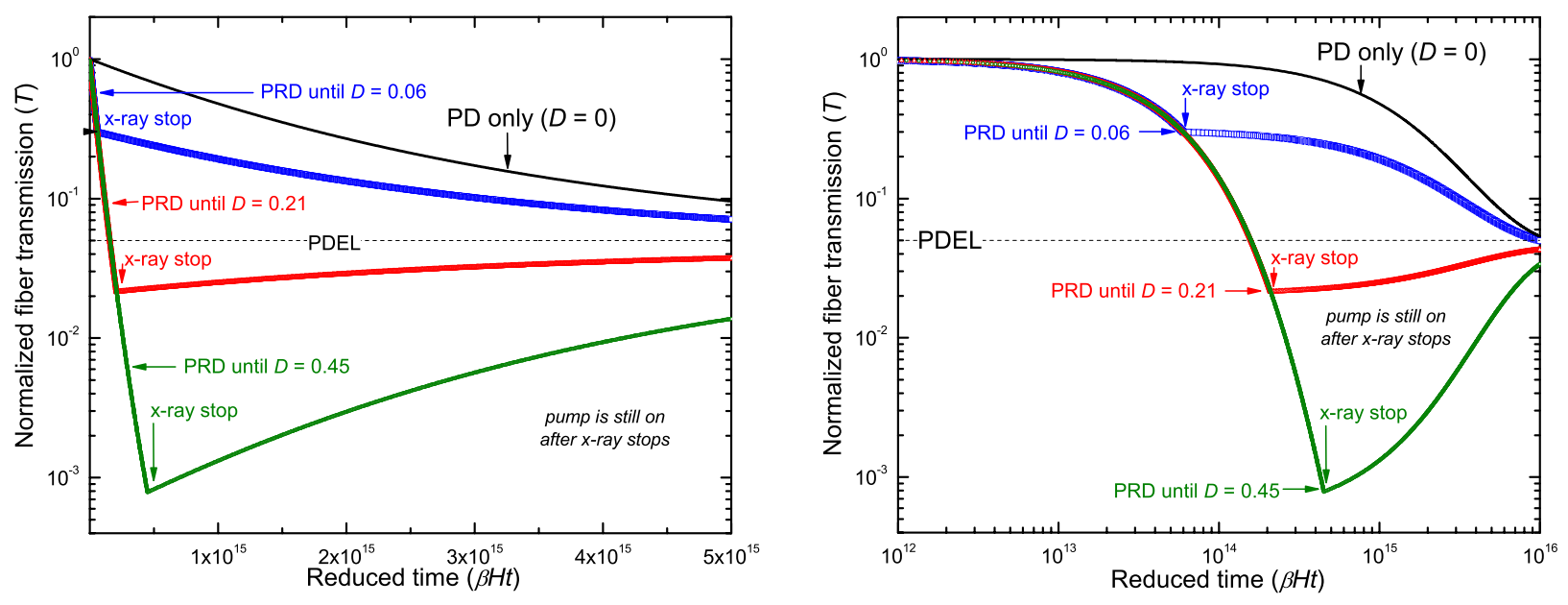

FIGURE 2. Simulated transmission decays at various reduced doses $D$. Same curves are plotted in linear (left) and log-log scales (right) to show the convergence towards the PDEL at long time 
The dose-dependent pump effect after PRD being established, we now turn to the characterization of the pump effect during PRD. In Fig. 3, similar to Fig. 3 in the Duchez's work, 2 ionizing doses ( $D=0.025$ and 0.4$)$ have been imparted with or without pump, i.e in PRD or RD conditions respectively, before leaving the pump acting alone. In order to illustrate the validity of the model in different situations, these calculations have been made with different parameters compared to those of Fig. 2. We still used $k=2, \alpha=1$ and $r=1$, but took: $\theta=10^{3}, x_{\text {inv }}=0.1, g_{0} /(\beta N)^{2}=$ $10^{-13}, N^{2} \sigma_{\mathrm{PD}} /\left(\tau N_{\mathrm{v}} \sigma_{\mathrm{PB}}\right)=0.625$ and $N^{3} \sigma_{\mathrm{PD}} /\left(\beta \tau^{3}\right)=1.6 \times 10^{-10}$. PD and PB kinetics are thus in similar proportions as in Fig. 2, but the PD and dose rates have been increased by a few thousands and a one hundred factor, respectively. Degradation is now plotted in terms of hole trap occupancy, proportional to the excess absorption coefficient $\Delta \alpha$ and directly comparable to data. At the end of the dose absorption, the pump slows-down darkening in PRD conditions, compared with RD conditions, at doses yielding a PRD level above PDEL $(D=0.4)$. Conversely, the pump enhances darkening at doses where the PRD level is below the PDEL $(D=0.025)$. This behavior is perfectly similar to that observed experimentally and this successful comparison provides a further support to the proposed model. In fact the pump has the same effect after as during PRD, depending on the ionizing dose and the PDEL.

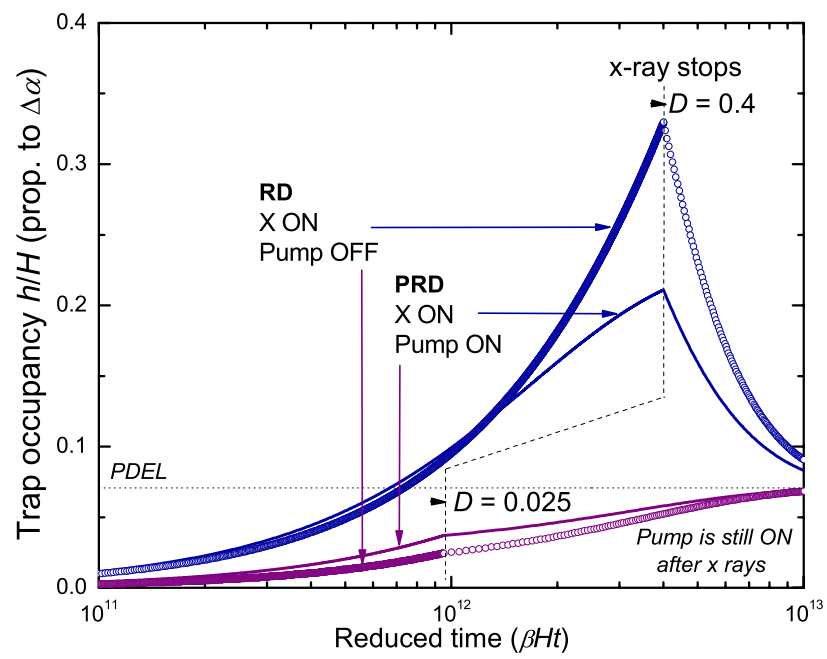

FIGURE 3. Simulated degradation under $\mathrm{X}$ rays in $\mathrm{RD}$ and $\mathrm{PRD}$ conditions, and post- $\mathrm{X}$-rays evolution due to the pump

At high doses, the PRD level can exceed the PDEL but it always reaches a plateau starting from a given dose due to the balancing PB action of the pump. This plateau is hereafter termed PRDEL (PRD equilibrium level). Examples of PRDEL can be seen in Fig. 4 (steady-state reached by dotted lines). Doses above the PRDEL threshold will not lead to further degradation of the YDF. The pump is therefore a natural mitigating agent at high doses. Moreover, increasing pump power and inversion will decrease the PRDEL. In fact, an increased inversion will raise the PDEL, according for instance to equations (5) or (6), but also the PB efficiency and so the "restoring force" towards the PDEL. As a consequence, the PRDEL will then decrease to get closer to the PDEL. In PD-resistant YDF, the PDEL will be low even for high inversion rates, and this radio-hardening effect related to the pump power will be enhanced. The PRDEL is of crucial interest: it sets the worst degradation level achievable in PRD conditions, so in operating conditions for the targeted applications. It is therefore the reference level to be optimized to improve the YDF resistance against PRD.

In addition to pump power and inversion, the PRDEL is very sensitive to the dose rate of the ionizing irradiation, because the stationary PRD level necessarily depends on the relative kinetics of pump- and radiation-induced processes (PD and PB vs RD rates). The effect of a decreasing dose rate is illustrated in Fig. 4, where simulations of the fractional irradiation experiments displayed in Fig. 4 of the Duchez's article are reproduced. Instead of considering a continuous irradiation, the dose was given in 1, 3 and 6 fractions; each of them being delivered at the same dose rate. A constant PB time was observed between each fraction. By increasing the fraction number, we well decrease the mean dose rate but, at the same time, we highlight clearly what happens in doing so. Indeed, sketching the low dose rate effect through such a "discrete" approach, i.e. with an increasing number of "shots" of decreasing duration, makes it easy to figure the continuous limit out (that of a constant dose rate). Calculations used the following parameters: $k=2, \alpha=1, r=1, \theta=10^{3}, x_{\mathrm{inv}}=0.1, g_{0} /(\beta N)^{2}=3.2 \times 10^{-13}, N^{2} \sigma_{\mathrm{PD}} /\left(\tau N_{\mathrm{v}} \sigma_{\mathrm{PB}}\right)=0.625$ and $N^{3} \sigma_{\mathrm{PD}} /\left(\beta \tau^{3}\right)=1.6 \times 10^{-11}, D=0.015$. Simulated plots are in very good agreement with data, at least qualitatively. 
Decay kinetics during PB stages is not correctly rendered actually, but this is not surprising in view of the simplicity of the model. Any improvement of this point would require a model refinement, e.g. by including a distribution of hole trapping levels. Fig. 4 also displays, in dashed lines, the PRD obtained for permanent irradiations, the constant dose rate being equal to the mean dose rate achieved in the 3- and 6-fraction irradiation runs (dashed lines). These continuous degradation curves well represent the mean trend of what we obtain with chopped irradiations, thus confirming that the fractional irradiation protocol is not misleading. Fig. 4 finally shows the PRD obtained for the same dose, but with a constant dose rate of one tenth and one thousandth times the 6-fraction mean dose rate. By exploring, through a validated simulation, such low dose rates that are not achievable in practice (too long irradiations), we demonstrate that the PRDEL decreases with increasing dose rate and that it saturates to its minimal value, equal to the PDEL, at sufficiently low dose rates. The explanation is straightforward: at very low dose rates, the RD build-up is very slow. As soon as the PDEL is exceeded due to RD, the pump has time to bleach the YDF "adiabatically" and therefore to bring the PRD level back to the PDEL.

The limit dose rate below which the PRDEL merges into the PDEL depends on the pump power. It increases with inversion. Indeed, high inversion rates, that already bring the PRDEL closer to the PDEL, require a lower dose rate decrease to make both stationary levels merge. As regards space-based applications, the very low space dose rate ensures that the PRDEL will not exceed the PDEL even for small pump power. Then, PD-resistant fibers will be PRD resistant throughout the mission. Because of the steady-state character of these levels with respect to the dose, this statement is true whatever the duration of the mission.

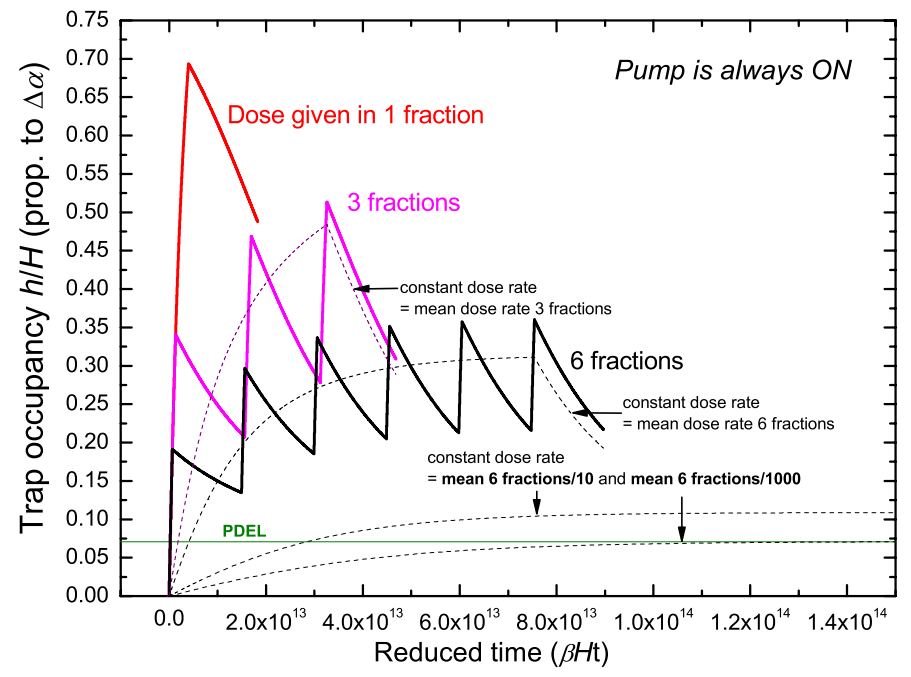

FIGURE 4. Simulation of PRD for a dose given in 1, 3 and 6 fractions and comparison with permanent irradiations at similar mean dose rates. This figure also illustrates that the PRDEL merge into the PDEL at very low dose rate.

\section{CONCLUSION}

This work proposes an original model of photo-darkening (PD), radiation-induced darkening (RD) and also of simultaneous photo-radio-darkening (PRD). The last term is used for convenience but is actually not really proper. The pump is not only responsible for PD, but also for photo-bleaching (PB), and PB effects are not marginal. Therefore the acronym PRD should be understood in the broad sense of "simultaneous pump- and radiation-induced effects".

The model is based on simple and clear physical mechanisms and, as a consequence, does not involve any semiempirical parameter. It has been cast into the framework of a trapping-recombination scheme and rationalized by related rate equations. Simulations have been conducted according to the same protocols as those followed for the experimental characterization of the interplay between PD and RD, as presented in the article by Duchez et al. (this proceeding). Calculated and measured data are always found to be in excellent qualitative agreement, either for pure PD or for PRD. The key to success in describing PD relies obviously on the supposed PD mechanisms (charge transfer of $\mathrm{Yb}^{3+}$ ions and photo-ionization of trapped-hole states, both triggered by cooperative energy releases from excited $\mathrm{Yb}^{3+}$ ions). The key to success in reproducing all of the PRD experimental features basically relies on the fact that PD and RD share the same trapped-hole centers, i.e. that the pump is capable of bleaching both PD and RD. 
Such successful comparisons against experimental data in a wide variety of situations strongly support our point of view.

Thanks to this validated model, the original observations of Duchez et al. are thoroughly and clearly explained. A very relevant physical framework is established to discuss, understand and predict the way YDF behave in radiation fields, in presence of the pump or not. If our minimal model is sufficient to get the basic trends, it is not able to predict accurately, or even correctly, actual PD or PRD kinetics. As already mentioned, possible improvements will notably have to consider a distribution of traps in the band gap, and therefore distributed values of the charge-transfer and photo-ionization cross-sections. Such refinements have to be sought, but they will be detrimental to the model universality. They will be highly dependent on the fiber composition and, probably, will have to be adapted for each YDF sample.

\section{ACKNOWLEDGMENTS}

This work has been conducted in the framework of the HAPoLO (098-AO11-2408) and PARADYSIO (ANR11-JS04-007) projects funded by the Airbus Group Foundation and the French Research National Agency (ANR). The authors gratefully acknowledge this support.

\section{REFERENCES}

1. I. Manek-Höninger, J. Boullet, T. Cardinal, F. Guillen, S. Ermeneux, M. Podgorski, R. Bello Doua and F. Salin, Opt. Express 15, 1606-1611 (2007).

2. A.D. Guzman-Chávez, A.V. Kir'yanov, Y.O. Barmenkov, N.N. Il'ichev, Laser Phys. Lett. 4, 734-739 (2007).

3. S. Jetschke, S. Unger, U. Röpke, and J. Kirchhof, Opt. Express 15, 14838-14843 (2007).

4. S. Yoo, C. Basu, A.J. Boyland, C. Sones, J. Nilsson, J.K. Sahu and D. Payne, Opt. Lett. 32, 1626-1628 (2007).

5. S. Jetschke and U. Röpke, Opt. Lett. 34, 109-111 (2009).

6. S. Jetschke, A. Schwuchow, S. Unger, M. Leich, M. Jäger, and J. Kirchhof, Opt. Mat. Express 3, 452-458 (2013).

7. F. Mady, M. Benabdesselam and W. Blanc, Opt. Lett. 35, 3541-3543 (2010).

8. F. Mady, M. Benabdesselam, Y. Mebrouk and B. Dussardier, Proceedings of the 11th international conference on Radiation Effects on Components and Systems (RADECS 2010), Längenfeld (Austria), 2010, Paper LN2, available at: http://www.radecs2010.ait.ac.at/index.php?link=programme.

9. S. Girard, Y. Ouerdane, B. Tortech, C. Marcandella, T. Robin, B. Cadier, J. Baggio, P. Paillet, V. FerletCavrois, A. Boukenter, J-P. Meunier, J.R. Schwank, M.R. Shaneyfelt, P.E. Dodd and E.W. Blackmore, IEEE Trans. Nucl. Sci. 56, 3293-3299 (2009).

10. S. Suzuki, H.A. McKay, X. Peng, L. Fu and L. Dong, Opt. Express 17, 9924-9932 (2009).

11. M. Engholm, P. Jelger, F. Laurell and L. Norin, Opt. Lett. 34, 1285-1287 (2009).

12. P. Jelger, M. Engholm, L. Norin and L. Faurell, J. Opt. Soc. Am. B 27, 338-342 (2010).

13. M. Engholm and L. Norin, Opt. Lett. 33, 1216 (2008).

14. S. Yoo, C. Basu, A.J. Boyland, C. Sones, J. Nilsson, J.K. Sahu and D. Payne, Opt. Lett. 33, 1217-1218 (2008).

15. M. Engholm, L. Norin and D. Aberg, Opt. Lett. 32, 3352-3354 (2007).

16. M. Engholm and L. Norin, Opt. Express 16, 1260-1268 (2008).

17. S. Rydberg and M. Engholm, Opt. Express 21, 6681-6688 (2013).

18. M. Leich, U. Röpke, S. Jetschke, S. Unger, V. Reichel, J. Khirchhof, Opt. Express 17, 12588-12593 (2009).

19. M. J. Söderlund, J.J. Montiel i Ponsoda, J. P. Koplow and S. Honkanen, Opt. Lett. 34, 2637-2639 (2009).

20. N. Li, S. Yoo, X. Yu, D. Jain and J.K. Sahu, IEEE Photon. Technol. Lett. 26, 115-118 (2014).

21. F. Mady, M. Benabdesselam, J-B. Duchez, Y. Mebrouk and S. Girard, IEEE Trans. Nucl. Sci. 60, 4341-3448 (2013). 